TRANSACTIONS OF THE

AMERICAN MATHEMATICAL SOCIETY

Volume 357, Number 1, Pages 397-407

S 0002-9947(04)03525-1

Article electronically published on July 22, 2004

\title{
CONVERGENCE OF DOUBLE FOURIER SERIES AND $W$-CLASSES
}

\author{
M. I. DYACHENKO AND D. WATERMAN
}

\begin{abstract}
The double Fourier series of functions of the generalized bounded variation class $\{n / \ln (n+1)\}^{*} B V$ are shown to be Pringsheim convergent everywhere. In a certain sense, this result cannot be improved. In general, functions of class $\Lambda^{*} B V$, defined here, have quadrant limits at every point and, for $f \in \Lambda^{*} B V$, there exist at most countable sets $P$ and $Q$ such that, for $x \notin P$ and $y \notin Q, f$ is continuous at $(x, y)$. It is shown that the previously studied class $\Lambda B V$ contains essentially discontinuous functions unless the sequence $\Lambda$ satisfies a strong condition.
\end{abstract}

\section{INTRODUCTION}

A remarkable variety of definitions of bounded variation have been given for functions of two variables. Here we will discuss generalizations of these definitions along the lines of the notion of $\Lambda$-bounded variation $(\Lambda B V)$ in one variable introduced by Waterman. He used it to extend the Dirichlet-Jordan theorem, and we will investigate the analogous problem for double Fourier series.

For an excellent discussion of $\Lambda B V$ and its relation to other generalizations of bounded variation, see Avdispahić 11. For applications to summability and Tauberian theorems, see 2811 .

Definition 1. Let $\Lambda=\left\{\lambda_{k}\right\}_{1}^{\infty}$ be a monotone nondecreasing sequence of positive numbers such that

$$
\sum_{1}^{\infty} \lambda_{k}^{-1}=\infty
$$

and let $Y$ denote the class of such sequences. A real function $f$ defined on an interval $[a, b]$ is said to be of $\Lambda$-bounded variation, $f \in \Lambda B V([a, b])$, if

$$
V_{\Lambda}(f ;[a, b])=\sup _{\mathcal{I}, n} \sum_{1}^{n} \frac{\left|f\left(I_{k}\right)\right|}{\lambda_{k}}=\sup _{\mathcal{I}, n} \sum_{1}^{n} \frac{\left|f\left(\beta_{k}\right)-f\left(\alpha_{k}\right)\right|}{\lambda_{k}}<\infty
$$

where $\mathcal{I}$ denotes the class of collections of nonoverlapping intervals $\left\{I_{k}=\left[\alpha_{k}, \beta_{k}\right] \subset\right.$ $[a, b], k=1, \ldots, n\}$.

Received by the editors March 17, 2003 and, in revised form, September 29, 2003.

2000 Mathematics Subject Classification. Primary 42B05, 26B30; Secondary 26B05.

Key words and phrases. Multiple Fourier series, generalized bounded variation, Waterman classes.

The first author gratefully acknowledges the support of RFFI grant N03-01-00080.

(C)2004 American Mathematical Society Reverts to public domain 28 years from publication 
Note that functions of $\Lambda$-bounded variation are bounded, have right and left limits at every point, and so their discontinuities are at most countable [9].

Function classes, whose definitions depend on the boundedness of sums of the absolute values of interval functions multiplied by weights from sequences such as $\Lambda$, have come to be known as $W$-classes.

In [7, 10], Waterman proved the following generalization of the Dirichlet-Jordan theorem.

Theorem A. If $f$ is a $2 \pi-$ periodic function, $H=\{n\}_{1}^{\infty}, T=[-\pi, \pi]$ and $f \in$ $H B V(T)$, then $S[f]$, the Fourier series of $f$, converges at every point and, if $I$ is a closed interval of points of continuity, then $S[f]$ converges uniformly on $I$. If $\Lambda B V \backslash H B V \neq \emptyset$, then there is an $f \in \Lambda B V(T)$ such that $S[f]$ diverges at a point.

A definition of $\Lambda B V$ for two variables which has been used by Saakjan [5] and Sablin [6] is as follows.

Definition 2. Let $\Lambda \in Y$ and let $f$ be a measurable function on the rectangle $A=[a, b] \times[c, d]$. Then $f \in \Lambda B V(A)$ if and only if

(1) $f(\cdot, c) \in \Lambda B V([a, b])$ and $f(a, \cdot) \in \Lambda B V([c, d])$, and

(2) if $\mathcal{I}_{1}$ and $\mathcal{I}_{2}$ are the sets of finite collections of nonoverlapping intervals $I_{k}=\left[\alpha_{k}, \beta_{k}\right]$ and $I_{j}=\left[\gamma_{j}, \delta_{j}\right]$ in $[a, b]$ and $[c, d]$ respectively and $f\left(I_{k} \times I_{j}\right)=$ $f\left(\alpha_{k}, \gamma_{j}\right)-f\left(\alpha_{k}, \delta_{j}\right)-f\left(\beta_{k}, \gamma_{j}\right)+f\left(\beta_{k}, \delta_{j}\right)$, then

$$
V_{\Lambda}(f ;[a, b])=\sup _{\mathcal{I}_{1}, \mathcal{I}_{2}} \sum_{k} \sum_{j} \frac{\left|f\left(I_{k} \times I_{j}\right)\right|}{\lambda_{k} \lambda_{j}}<\infty .
$$

Remark 1. If $\lambda_{k} \equiv 1$, or what is the same, $\lambda_{k}=O(1), \Lambda B V(A)$ is the set of functions of Hardy-Krause bounded variation on $A$.

It is clear that the functions of $\Lambda B V(A)$ are bounded, but the question of continuity is more complicated than in the case of functions of one variable. Dyachenko [3] has proved the following theorem.

Theorem B. The following conditions are equivalent:

(i) for any $f \in \Lambda B V\left(T^{2}\right)$ there exist two at most countable subsets $A$ and $B$ of $T$ such that $f$ is continuous at every point $(x, y) \in T^{2}$ such that $x \notin A$ and $y \notin B$;

(ii) for any $f \in \Lambda B V\left(T^{2}\right)$ and any $\left(x_{0}, y_{0}\right) \in T^{2}, \lim f(x, y)$ exists as $(x, y) \rightarrow$ $\left(x_{0}, y_{0}\right)$ in each of the open coordinate quadrants;

(iii) $\sum_{1}^{\infty} \lambda_{k}^{-2}=\infty$.

(The third condition will be called Condition $(*)$.)

Thus we see, for example, that the characteristic function of the triangle $B=$ $\left\{(x, y) \in[0,1]^{2}, 0 \leq y \leq 1-x\right\}$ is in $\Lambda B V\left([0,1]^{2}\right)$ only if Condition $(*)$ does not hold.

If $\Lambda$ does not satisfy (iii), the requirement of measurability cannot be omitted from Definition 2, for if it were, $\Lambda B V(A)$ would include functions not Lebesgue measurable. Even under the assumption of measurability, we show in Section 2 that $\Lambda B V(A)$ contains an everywhere discontinuous function and, moreover, a function $f$ such that if $g=f$ a.e., then $g$ is a.e. discontinuous. 
We will consider the Pringsheim convergence of double Fourier series. If $f \in$ $L\left(T^{2}\right)$ is $2 \pi$-periodic in each variable, then

$$
S[f]=\sum_{m, n} a_{m n} e^{i(m x+n y)}
$$

is its Fourier series, where

$$
a_{m n}=a_{m n}(f)=\frac{1}{(2 \pi)^{2}} \int_{T^{2}} f(x, y) e^{-i(m x+n y)} d x d y .
$$

The rectangular partial sums of this series are

$$
S_{N_{1} N_{2}}(f ; x, y)=\sum_{\left|k_{1}\right| \leq N_{1}} \sum_{\left|k_{2}\right| \leq N_{2}} a_{k_{1} k_{2}} e^{i\left(k_{1} x+k_{2} y\right)}
$$

with $N_{1}, N_{2} \geq 0$. If $N_{1}=N_{2}$, these are called square sums. If

$$
S_{N_{1} N_{2}}(f ; x, y) \rightarrow \alpha \text { as } \min \left(N_{1}, N_{2}\right) \rightarrow \infty,
$$

we say that the Fourier series of $f$ converges to $\alpha$ at $(x, y)$ in the Pringsheim sense.

A.A. Saakyan [5] has shown

Theorem C. If $f \in H B V\left(T^{2}\right)$, then the rectangular partial sums of $S[f]$ are uniformly bounded, and, if for $\left(x_{o}, y_{0}\right) \in T^{2}$ the limits of $f(x, y)$ exist as $(x, y) \rightarrow$ $\left(x_{o}, y_{0}\right)$ in each of the open coordinate quadrants, then $S[f]$ converges (Pringsheim) to the arithmetic mean of these limits.

This result has been generalized to higher dimensions by A.I. Sablin 6 .

As we shall see in $\S 2$, an $f \in H B V\left(T^{2}\right)$ need not have a point of continuity. For such a function, Theorem $\mathrm{C}$ is inapplicable.

We shall define another $W$-class such that functions of this class are continuous a.e., and prove that a theorem analogous to Theorem $\mathrm{C}$ holds for this class.

Definition 3. Let $\Lambda \in Y$ and let $f$ be a real function on $A=[a, b] \times[c, d]$. We say $f \in \Lambda^{*} B V(A)$ if

(i) $f(\cdot, c) \in \Lambda B V([a, b])$ and $f(a, \cdot) \in \Lambda B V([c, d])$

and, if $\Gamma$ is the set of finite collections of nonoverlapping rectangles $A_{k}=\left[\alpha_{k}, \beta_{k}\right] \times$ $\left[\gamma_{k}, \delta_{k}\right] \subset A$ and $f\left(A_{k}\right)=f\left(\alpha_{k}, \gamma_{k}\right)-f\left(\alpha_{k}, \delta_{k}\right)-f\left(\beta_{k}, \gamma_{k}\right)+f\left(\beta_{k}, \delta_{k}\right)$, then

(ii) $V_{\Lambda}^{*}(f ; A)=\sup _{\Gamma} \sum_{k} \frac{\left|f\left(A_{k}\right)\right|}{\lambda_{k}}<\infty$.

For $f \in \Lambda^{*} B V(A)$ we set

(iii) $\|f\|_{\Lambda^{*}}=\|f\|_{\Lambda^{*}(A)}=|f(a, c)|+V_{\Lambda}(f(\cdot, c))+V_{\Lambda}(f(a, \cdot))+V_{\Lambda}^{*}(f ; A)$.

Remark 2. Note that if $f \in \Lambda^{*} B V(A)$, then

$$
V_{\Lambda}(f(\cdot, y) ;[a, b]) \leq V_{\Lambda}^{*}(f ; A)+V_{\Lambda}(f(\cdot, c) ;[a, b])
$$

for every $y \in[c, d]$. The analogous result holds for the $\Lambda$-variation of the restriction of $f$ to the vertical segments.

In $\S 3$ we shall prove

Theorem 1. Let $\Lambda \in Y$ and $A=[a, b] \times[c, d]$. Then, for any $f \in \Lambda^{*} B V(A)$,

(i) there exist at most countable sets $P \subset[a, b]$ and $Q \subset[c, d]$ such that $f$ is continuous at every $(x, y) \in A$ such that $x \notin P$ and $y \notin Q$; and

(ii) at every point $\left(x_{0}, y_{0}\right) \in A, \lim _{(x, y) \rightarrow\left(x_{0}, y_{0}\right)} f(x, y)$ exists in each open coordinate quadrant. 
In that section we also discuss the relation between $\Lambda^{*} B V(A)$ and $\Lambda B V(A)$.

In $\S 4$ we study the convergence of Fourier series of functions of class $\Lambda^{*} B V(A)$, and prove

Theorem 2. Let $f$ be a real function on $\mathbb{R}^{2}$ which is $2 \pi$-periodic in each variable and is in $\Lambda^{*} B V\left(T^{2}\right)$ with $\Lambda=\left\{\frac{n}{\ln (n+1)}\right\}$. Then the rectangular partial sums of $S[f]$ are uniformly bounded and converge at each point to the arithmetic mean of the quadrant limits.

We also show that, in a certain sense, this result cannot be improved.

Theorem 3. Let $\Lambda=\left\{\frac{n}{\ln (n+1)} \xi_{n}\right\} \in Y$, where $\xi_{n} \uparrow \infty$ as $n \rightarrow \infty$. Then there exists a function $f \in \Lambda^{*} B V\left(T^{2}\right)$ such that the square partial sums of its Fourier series diverge unboundedly at $(0,0)$.

\section{Discontinuous functions in $W$-Classes}

We shall require the following lemmas.

Lemma 1. Let $\Lambda \in Y$ be such that Condition $(*)$ does not hold (i.e., $\sum \lambda_{k}^{-2}<\infty$ ) and let $A=[a, b] \times[c, d]$ be a nondegenerate interval. Suppose $E \subset A$ has a connected intersection with every horizontal and vertical line. Then $\chi_{E}$, the characteristic function of $E$, is in $\Lambda B V(A)$ and $V_{\Lambda}\left(\chi_{E} ; A\right)<C<\infty$, where $C$ is an absolute constant.

Proof. Let $\left\{I_{k}\right\}_{1}^{n}=\left\{\left[\alpha_{k}, \beta_{k}\right]\right\}$ in $[a, b]$ and $\left\{J_{r}\right\}_{1}^{m}=\left\{\left[\gamma_{r}, \delta_{r}\right]\right\}$ in $[c, d]$ be two collections of nonoverlapping intervals. Then, for each $k=1,2, \ldots, n$, in the sum

$$
S=\sum_{k=1}^{n} \sum_{r=1}^{m}\left|\chi_{E}\left(I_{k} \times J_{r}\right)\right| / \lambda_{k} \lambda_{r}
$$

there are at most four different $r_{k, j}$ for which $\left|\chi_{E}\left(I_{k} \times J_{r_{k, j}}\right)\right| \neq 0$, and in these cases it is either 1 or 2 . Let

$$
S_{j}=\sum_{k=1}^{n} \frac{\left|\chi_{E}\left(I_{k} \times J_{r_{k, j}}\right)\right|}{\lambda_{k} \lambda_{r_{k, j}}}, \quad \text { for } j=1,2,3,4 .
$$

Then $S=S_{1}+S_{2}+S_{3}+S_{4}$, and, as each $j$ can be associated with at most four $r_{k, j}$, we have

$$
S_{j} \leq 2 \sum_{k=1}^{n} \frac{1}{\lambda_{k} \lambda_{r_{k, j}}} \leq \sum_{k=1}^{n} \frac{1}{\lambda_{k}^{2}}+\sum_{k=1}^{n} \frac{1}{\lambda_{r_{k, j}}^{2}} \leq 5 \sum_{k=1}^{n} \frac{1}{\lambda_{k}^{2}}<C<\infty
$$

and, since the one-dimensional $\Lambda$-variation of $\chi_{E}$ on the edges of $A$ is at most $2 / \lambda_{1}$, Lemma 1 is established.

Lemma 2. $A=[a, b] \times[c, d]$ be a nondegenerate interval. There is a sequence of closed rectangles $\left\{A_{i}=I_{i} \times J_{i}\right\}$ in $A$ with $I_{i} \cap I_{j}=\emptyset$ and $J_{i} \cap J_{j}=\emptyset$ for $i \neq j$, with

$$
\sum_{i=1}^{\infty}\left|I_{i}\right|<(b-a) / 4 \text { and } \sum_{i=1}^{\infty}\left|J_{i}\right|<(d-c) / 4,
$$

such that every neighborhood of each point of the following contains some $A_{i}$ :

$$
B=A \backslash \bigcup_{i=1}^{\infty}\left(\left(I_{i} \times[c, d]\right) \cup\left([a, b] \times J_{i}\right)\right) .
$$


Proof. Choose $n_{1}=1$ and consider the rectangles

$$
E_{k, r, 1}=\left[a+\frac{(b-a)(k-1)}{2^{n_{1}}}, a+\frac{(b-a) k}{2^{n_{1}}}\right] \times\left[c+\frac{(d-c)(r-1)}{2^{n_{1}}}, c+\frac{(d-c) r}{2^{n_{1}}}\right],
$$

where $k, r=1,2 \ldots, 2^{n_{1}}$.

Choose an integer $n_{2}>2 n_{1}+3$ and, for each choice of $k$ and $r, 1 \leq k, r \leq 2^{n_{1}}$, choose a rectangle

$$
\begin{gathered}
E_{k, r, 1}^{\prime}=\left[a_{k, r, 1}, b_{k, r, 1}\right] \times\left[c_{k, r, 1}, d_{k, r, 1}\right] \\
=\left[a+\frac{(b-a)\left(l_{k}-1\right)}{2^{n_{2}}}, a+\frac{(b-a) l_{k}}{2^{n_{2}}}\right] \times\left[c+\frac{(d-c)\left(s_{r}-1\right)}{2^{n_{2}}}, c+\frac{(d-c) s_{r}}{2^{n_{2}}}\right] \subset E_{k, r, 1}
\end{gathered}
$$

so that the projections of the chosen rectangles on the coordinate axes do not touch. Clearly

$$
\sum_{k, r=1}^{2^{n_{1}}}\left(b_{k, r, 1}-a_{k, r, 1}\right)<\frac{b-a}{8} \text { and } \sum_{k, r=1}^{2^{n_{1}}}\left(d_{k, r, 1}-c_{k, r, 1}\right)<\frac{d-c}{8} .
$$

Next we consider the rectangles

$$
E_{k, r, 2}=\left[a+\frac{(b-a)(k-1)}{2^{n_{2}}}, a+\frac{(b-a) k}{2^{n_{2}}}\right] \times\left[c+\frac{(d-c)(r-1)}{2^{n_{2}}}, c+\frac{(d-c) r}{2^{n_{2}}}\right],
$$

where $k$ and $r$ are chosen, $1 \leq k, r \leq 2^{n_{2}}$, so that

$$
E_{k, r, 2} \cap \bigcup_{k, r=1}^{2^{n_{1}}}\left(\left[a_{k, r, 1}, b_{k, r, 1}\right] \times[c, d]\right) \cup\left([a, b] \times\left[c_{k, r, 1}, d_{k, r, 1}\right]\right)=\emptyset .
$$

We then choose an integer $n_{3}>2 n_{2}+4$ and, for each $k$ and $r, 1 \leq k, r \leq 2^{n_{2}}$, just chosen, we choose a rectangle

$$
\begin{aligned}
& E_{k, r, 2}^{\prime}=\left[a_{k, r, 2}, b_{k, r, 2}\right] \times\left[c_{k, r, 2}, d_{k, r, 2}\right] \\
& =\left[a+\frac{(b-a)\left(l_{k}-1\right)}{2^{n_{3}}}, a+\frac{(b-a) l_{k}}{2^{n_{3}}}\right] \times\left[c+\frac{(d-c)\left(s_{r}-1\right)}{2^{n_{3}}}, c+\frac{(d-c) s_{r}}{2^{n_{3}}}\right] \\
& \subset E_{k, r, 2}
\end{aligned}
$$

so that the projections of the chosen rectangles on the coordinate axes do not touch. Then

$$
\sum_{k, r}\left(b_{k, r, 2}-a_{k, r, 2}\right)<\frac{b-a}{16} \text { and } \sum_{k, r}\left(d_{k, r, 2}-c_{k, r, 2}\right)<\frac{d-c}{16} .
$$

Proceeding inductively, we can define $E_{k, r, j}^{\prime}, j=1,2, \ldots$, choosing $n_{j+1}>2 n_{j}+$ $j+2$ at each step, and, renumbering the $E_{k, r, j}^{\prime}$ as we wish, $\left\{A_{i}\right\}=\left\{E_{k, r, j}^{\prime}\right\}$ is the required sequence of intervals.

We turn now to the principal results of this section.

Proposition 1. Suppose $\Lambda \in Y, A=[a, b] \times[c, d]$ is a nondegenerate rectangle and Condition (*) does not hold. Then there exists an $f \in \Lambda B V(A)$ which is everywhere discontinuous. 
Proof. Let $P$ and $Q$ be the sets of rationals in $[a, b]$ and $[c, d]$ respectively. Divide the rectangle $A$ into four quarters by passing lines parallel to the axes through the midpoints of the sides. In each of the rectangles $A_{i}, i=1,2,3,4$, thus formed, we select $\left(p_{i}, q_{i}\right), p_{i} \in P, q_{i} \in Q$, so that the $\left\{p_{i}\right\}$ and $\left\{q_{i}\right\}$ are distinct. Now we can quarter each $A_{i}$ and choose one point in each sixteenth not yet containing a chosen point to form $\left\{\left(p_{i}, q_{i}\right)\right\}_{5}^{16}, p_{i} \in P, q_{i} \in Q$, so that $\left\{p_{i}\right\}_{1}^{16}$ and $\left\{q_{i}\right\}_{1}^{16}$ are sets of distinct points. Proceeding inductively, we obtain a dense set of points $E=\left\{\left(p_{i}, q_{i}\right)\right\}$ such that $p_{i} \neq p_{j}$ and $q_{i} \neq q_{j}$ when $i \neq j$. Thus any line parallel to an axis meets $E$ in at most one point and, by Lemma $1, \chi_{E} \in \Lambda B V(A)$.

The function we have constructed in Proposition 1 is everywhere discontinuous but is equivalent to the function identically equal to zero. The next result shows that in a class $\Lambda B V(A)$ in which Condition (*) does not hold there exist essentially discontinuous functions.

Proposition 2. Suppose $\Lambda \in Y, A=[a, b] \times[c, d]$ is a nondegenerate rectangle and Condition (*) does not hold. Then there is an $f \in \Lambda B V(A)$ such that $g=f$ a.e. implies that $g$ is a.e. discontinuous.

Proof. Apply Lemma 2 to form the sequence $\left\{A_{i}\right\}$ and the set $B_{1}=B$. If $F_{1}=\bigcup_{1}^{\infty} A_{i}$ and $f_{1}=\chi_{F_{1}}$, we see that $V_{\Lambda}\left(f_{1}\right)=C<\infty$, and we observe that $\left|A \backslash B_{1}\right|<|A| / 2$. The set $A \backslash B_{1}$ can be divided into rectangles $\left\{D_{i}\right\}_{i=1}^{\infty}$ in the natural way. By applying Lemma 2 to each of the rectangles $D_{i}$, we obtain for each $i$ a sequence $\left\{A_{i j}\right\}_{j=1}^{\infty} \subset D_{i}$ with similar properties. Let $F_{2, i}=\bigcup_{j=1}^{\infty} A_{i, j}$ and $f_{2, i}=\chi_{F_{2, i}}, i=$ $1,2, \ldots$. Note that $V_{\Lambda}\left(f_{2, i}\right) \leq C$ for every $i$. If $A_{i, j}=\left[a_{i, j}, b_{i, j}\right] \times\left[c_{i, j}, d_{i, j}\right]$, set

$$
B_{2, i}=D_{i} \backslash \bigcup_{j=1}^{\infty}\left(\left(\left[a_{i, j}, b_{i, j}\right] \times[c, d]\right) \cup\left([a, b] \times\left[c_{i, j}, d_{i, j}\right]\right)\right), \quad i=1,2, \ldots .
$$

Let us replace $i$ by the symbol $i_{1}$. At the third stage we obtain sets $B_{3, i_{1}, i_{2}}$, and as the induction proceeds we obtain sets $B_{r, i_{1}, \ldots i_{r-1}}$. Let

$$
U=B_{1} \cup\left(\bigcup_{r=2}^{\infty}\left(\bigcup_{i_{1}, \ldots i_{r-1}=1}^{\infty} B_{r, i_{1}, \ldots i_{r-1}}\right)\right) ;
$$

then $|A \backslash U|=0$. We continue inductively to obtain functions $f_{1},\left\{f_{2, i_{1}}\right\}_{i_{1}=1}^{\infty}, \ldots$, $\left\{f_{r, i_{1}, \cdots i_{r-1}}\right\}_{i_{1}, \cdots i_{r-1}=1}^{\infty}, \ldots$, and we renumber these functions to form $\left\{h_{k}\right\}_{k=1}^{\infty}$. Now, letting

$$
f=\sum_{k=1}^{\infty} 3^{-k} h_{k}
$$

we have $f \in \Lambda B V(A)$.

Let $g=f$ a.e. and let $E$ be the set of points in $A$ at which $g$ and $f$ are equal. Clearly $E$ is dense in $A$. We write $\omega(f ;(x, y) ; E)$ for the oscillation of $f$ at $(x, y)$ over the set $E$ and $\omega(g ;(x, y) ; A)$ for the oscillation of $g$ at $(x, y)$ over the set $A$. Note that

$$
\omega(g ;(x, y) ; A) \geq \omega(f ;(x, y) ; E) .
$$

Consider a point $(x, y) \in U$. Then $(x, y)$ is in some $B_{r, i_{1}, \ldots i_{r-1}}$. If $k$ is such that $h_{k}=f_{r, i_{1}, \ldots i_{r-1}}$, then $\omega\left(h_{k} ;(x, y) ; E\right)=1$. Note that, since $h_{l}(A)=\{0,1\}$, for every 
$(s, t) \in A$ and every $l$, we have either

$$
\omega\left(h_{l} ;(s, t) ; E\right)=0 \text { or } \omega\left(h_{l} ;(s, t) ; E\right)=1 .
$$

Let

$$
k_{0}=k_{0}(x, y)=\min \left\{l: \omega\left(h_{l} ;(x, y) ; E\right)=1\right\} .
$$

Then

$$
\omega(f ;(x, y) ; E) \geq 3^{-k_{0}}-\sum_{l=k_{0}+1}^{\infty} 3^{-l} \omega\left(h_{l} ;(x, y) ; E\right) \geq 3^{-k_{0}}-\sum_{l=k_{0}+1}^{\infty} 3^{-l} \geq 3^{-k_{0}-1},
$$

implying that $g$ is discontinuous at $(x, y)$.

\section{Continuity properties of Functions of $\Lambda^{*} B V$}

We now turn our attention to the proof of Theorem 1.

Proof. Suppose there are infinitely many points $\left(x_{i}, y_{i}\right)$ such that $x_{i} \neq x_{j}$ and $y_{i} \neq y_{j}$ for $i \neq j$, at which the oscillation of $f$ exceeds $1 / k, k$ a natural number. For a natural number $N$ we can find points $\left(\alpha_{i}, \beta_{i}\right)$ and $\left(\gamma_{i}, \delta_{i}\right), i=1,2, \ldots, N$, such that $f\left(\alpha_{i}, \beta_{i}\right)-f\left(\gamma_{i}, \delta_{i}\right)>1 / k$ and the sequences $\alpha_{1}, \gamma_{1}, \alpha_{2}, \gamma_{2}, \alpha_{3}, \ldots$ and $\beta_{1}, \delta_{1}, \beta_{2}, \delta_{2}, \beta_{3}, \ldots$ are strictly monotone. We will assume them to be increasing; the other cases are handled similarly.

We have

$$
\begin{aligned}
S_{1} & =\sum_{i=0}^{N} \frac{\left|f\left(\alpha_{i}, \delta_{i}\right)-f\left(\gamma_{i}, \delta_{i}\right)\right|}{\lambda_{i}} \\
& \leq \sum_{i=0}^{N} \frac{\left|f\left(\left(\alpha_{i}, \gamma_{i}\right) \times\left(c, \delta_{i}\right)\right)\right|}{\lambda_{i}}+\sum_{i=0}^{N} \frac{\left|f\left(\alpha_{i}, c\right)-f\left(\gamma_{i}, c\right)\right|}{\lambda_{i}} \leq\|f\|_{\Lambda^{*}}
\end{aligned}
$$

and, in a similar fashion,

$$
S_{2}=\sum_{i=0}^{N} \frac{\left|f\left(\left(\gamma_{i}, \beta_{i}\right)-f\left(\gamma_{i}, \delta_{i}\right)\right)\right|}{\lambda_{i}} \leq\|f\|_{\Lambda^{*}}^{*} .
$$

Thus

$$
\begin{aligned}
V_{\Lambda^{*}}(f ; A) & \geq \sum_{i=0}^{N} \frac{\left|f\left(\left(\alpha_{i}, \gamma_{i}\right) \times\left(\beta_{i}, \delta_{i}\right)\right)\right|}{\lambda_{i}} \\
& \geq \sum_{i=0}^{N} \frac{\left|f\left(\alpha_{i}, \beta_{i}\right)-f\left(\gamma_{i}, \delta_{i}\right)\right|}{\lambda_{i}}-S_{1}-S_{2} \\
& \geq \frac{1}{k} \sum_{i=0}^{N} \frac{1}{\lambda_{i}}-2\|f\|_{\Lambda^{*}},
\end{aligned}
$$

which is false for $N$ sufficiently large. Thus all points at which $f$ has an oscillation greater than $1 / k$ lie on a finite number of lines parallel to the axes, which establishes the first part of the theorem.

To establish the second part of Theorem 1, we assume that there is a point $p \in A$ such that $f(x, y)$ does not have a limit as $(x, y) \rightarrow p$ within an open coordinate quadrant with vertex $p$. Without loss of generality we may assume that $p=(0,0)$ and the quadrant is $\{(x, y): x>0, y>0\}$. 
Then there is an $\varepsilon>0$ such that, for every $\delta>0$, in every square $(0, \delta)^{2}$ the oscillation of $f$ is greater than $\varepsilon$. Choose $s, t>0$. Then, since $f$ is in $\Lambda B V$ in each variable separately, $\lim _{y \downarrow 0} f(s, y)$ and $\lim _{x \downarrow 0} f(x, t)$ exist. Choose $\delta>0$ so that the oscillations of $f(s, y)$ and $f(x, t)$ on $0<y<\delta$ and $0<x<\delta$ respectively are less than $\varepsilon / 8$. Now choose points $\left(x_{1}, y_{1}\right)$ and $\left(x_{2}, y_{2}\right)$ in $(0, \delta)^{2}$ so that

$$
\left|f\left(x_{1}, y_{1}\right)-f\left(x_{2}, y_{2}\right)\right|>\varepsilon / 2 \text {. }
$$

Then, letting $P=f(s, t)-f\left(s, y_{1}\right)-f\left(x_{1}, t\right)+f\left(x_{1}, y_{1}\right)$ and $Q=f(s, t)-f\left(s, y_{2}\right)-$ $f\left(x_{2}, t\right)+f\left(x_{2}, y_{2}\right)$, we have

$$
\begin{aligned}
|P-Q| & \geq\left|f\left(x_{1}, y_{1}\right)-f\left(x_{2}, y_{2}\right)\right|-\left|f\left(x_{1}, t\right)-f\left(x_{2}, t\right)\right|-\left|f\left(s, y_{1}\right)-f\left(s, y_{2}\right)\right| \\
& >\varepsilon / 2-\varepsilon / 8-\varepsilon / 8=\varepsilon / 4,
\end{aligned}
$$

so that at least one of $|P|$ and $|Q|$ exceeds $\varepsilon / 8$, and so we have obtained a rectangle $A_{1} \in(0, \delta)^{2}$ for which $\left|f\left(A_{1}\right)\right|>\varepsilon / 8$. By choosing our points $(s, t),\left(x_{1}, y_{1}\right)$ and $\left(x_{2}, y_{2}\right)$ sufficiently close to the origin, we can repeat this process to obtain a rectangle $A_{2}$ which does not overlap $A_{1}$ for which $\left|f\left(A_{2}\right)\right|>\varepsilon / 8$. Thus we can form a sequence $\left\{A_{n}\right\}$ of nonoverlapping intervals in $(0, \delta)^{2}$ with $\left|f\left(A_{n}\right)\right|>\varepsilon / 8$. Then

$$
\sum_{i=0}^{N} \frac{\left|f\left(A_{k}\right)\right|}{\lambda_{k}}>\frac{\varepsilon}{8} \sum_{i=0}^{N} \frac{1}{\lambda_{k}} \rightarrow \infty \text { as } N \rightarrow \infty,
$$

contradicting our assumption that $f \in \Lambda^{*} B V(A)$, and completing the proof of Theorem 1.

It is natural to ask how the classes $\Lambda B V$ and $\Lambda^{*} B V$ are related. This is by no means obvious, although they are clearly the same if $\left\{\lambda_{i}\right\}$ is bounded. There is no loss of generality in assuming the rectangle $A$ to be $[0,1]^{2}$.

Proposition 3. If $\Lambda \in Y$ is an unbounded sequence, then $\Lambda B V \backslash \Lambda^{*} B V \neq \emptyset$.

Proof. First we consider the case where $\sum_{1}^{\infty} \lambda_{i}^{-2}<\infty$ and consider $\chi_{E}$, where

$$
E=\left\{(x, y) \in[0,1]^{2}, y \leq 1-x\right\} \text {. }
$$

Lemma 1 implies that $\chi_{E} \in \Lambda B V$, but from Theorem 1 we have $\chi_{E} \notin \Lambda^{*} B V$.

Now assume that Condition (*) holds and $\lambda_{i} \rightarrow \infty$ as $i \rightarrow \infty$. We choose $\alpha_{i} \searrow 0$ so that

$$
\sum_{i} \frac{\alpha_{i}}{\lambda_{i}}=\infty \text { and } \sum_{i} \frac{\alpha_{i}}{\lambda_{i}^{2}}<\infty
$$

Let

$$
f=\sum_{1}^{\infty} \alpha_{n} \chi_{E_{n}}, \quad \text { where } E_{n}=\left[\frac{1}{2 n}, \frac{1}{2 n-1}\right]^{2} .
$$

The rectangles $A_{n}=[2 /(4 n+1), 1 / 2 n]^{2}$ are pairwise disjoint and $\left|f\left(A_{n}\right)\right|=\alpha_{n}$. Hence

$$
\sum_{i=1}^{N} \frac{\left|f\left(A_{i}\right)\right|}{\lambda_{i}}=\sum_{i=1}^{N} \frac{\alpha_{i}}{\lambda_{i}} \rightarrow \infty \quad \text { as } N \rightarrow \infty,
$$

implying $f \notin \Lambda^{*} B V$.

Clearly, $f(\cdot, 0)$ and $f(0, \cdot)$ are in $\Lambda B V([0.1])$. Suppose $\left\{I_{i}\right\}_{1}^{N}$ and $\left\{J_{i}\right\}_{1}^{N}$ are collections of nonoverlapping intervals in $[0,1]$. For each $I_{i}$, there are no more than four values of $j$ such that $f\left(I_{i} \times J_{j}\right) \neq 0$. Let $j(i)$ denote the smallest. Let $k(i)$ 
be the smallest of the indices of $E_{n}$ such that $\chi_{E_{n}}\left(I_{i} \times J_{j}\right) \neq 0$ for some $j$. Then $\left|f\left(I_{i} \times J_{j}\right)\right| \leq 2 \alpha_{k(i)}$. Also, each $k$ can appear no more than twice as a $k(i)$ and each $j$ can appear no more than twice as a $j(i)$. Thus

$S=\sum_{1, j=1}^{N} \frac{\left|f\left(I_{i} \times J_{j}\right)\right|}{\lambda_{i} \lambda_{j}} \leq 8 \sum_{i=1}^{N} \frac{\alpha_{k(i)}}{\lambda_{i} \lambda_{j(i)}} \leq 8\left(\sum_{i=1}^{N} \frac{\alpha_{k(i)}}{\lambda_{i}^{2}}\right)^{\frac{1}{2}}\left(\sum_{i=1}^{N} \frac{\alpha_{k(i)}}{\lambda_{j(i)}^{2}}\right)^{\frac{1}{2}} \leq 32 \sum_{i=1}^{N} \frac{\alpha_{i}}{\lambda_{i}^{2}}$,

which is bounded above independently of $N$ and the choice of $\left\{I_{i}\right\}$ and $\left\{J_{j}\right\}$. Thus $f \in \Lambda B V$.

Proposition 4. If $A=[a, b] \times[c, d], 0<\alpha \leq 1$, and $\Lambda_{\alpha}=\left\{n^{\alpha}\right\}_{n=1}^{\infty}$, then

$$
\Lambda_{\alpha}^{*} B V \backslash \Lambda_{\alpha} B V \neq \emptyset \text {. }
$$

Proof. We will assume once again the $A=[0,1]^{2}$. Let

$$
f_{n}=\sum_{k=1}^{n} \sum_{1 \leq l \leq n / k}(-1)^{k+l} \chi_{\left[\frac{k-1}{n}, \frac{k}{n}\right) \times\left[\frac{l-1}{n}, \frac{l}{n}\right)}
$$

$C$ will denote a constant, not necessarily the same at each occurrence, and $C_{\alpha}$ a constant depending on $\alpha$. The number of terms in the sum defining $f_{n}$ is not greater than $n \ln (n+1)$, so for $\alpha \in(0,1)$,

$$
V_{\Lambda_{\alpha}^{*}}\left(f_{n}\right) \leq C \sum_{r=1}^{n \ln (n+1)} r^{-\alpha} \leq C_{\alpha}(n \ln (n+1))^{1-\alpha} .
$$

Similarly,

$$
V_{\Lambda_{1}^{*}}\left(f_{n}\right) \leq C \ln (n+1) .
$$

On the other hand, for $\alpha \in(0,1)$,

$$
V_{\Lambda_{\alpha}}\left(f_{n}\right) \geq C \sum_{k=1}^{n} k^{-\alpha}\left(\sum_{j=1}^{n / k} j^{-\alpha}\right) \geq C_{\alpha} \sum_{k=1}^{n} k^{-\alpha}\left(\frac{n}{k}\right)^{1-\alpha} \geq C_{\alpha} n^{1-\alpha} \ln (n+1),
$$

and similarly,

$$
V_{\Lambda_{1}}\left(f_{n}\right) \geq C(\ln (n+1))^{2} .
$$

Thus, if

$$
f(x, y)=\sum_{k=1}^{\infty} a_{k} f_{n_{k}}\left(2^{k} x-1,2^{k} y-1\right),
$$

where the sequence of coefficients $\left\{a_{k}\right\}$ and the increasing sequence of natural numbers $\left\{n_{k}\right\}$ are appropriately chosen, we will have

$$
f \in \Lambda_{\alpha}^{*} B V \backslash \Lambda_{\alpha} B V .
$$

The general problem remains open. 


\section{Convergence of Double Fourier Series}

Theorem 2 follows immediately from Theorem $\mathrm{C}$ and the following lemma.

Lemma 3. Let $\Lambda=\left\{\frac{n}{\ln (n+1)}\right\}_{n=1}^{\infty}, A=[a, b] \times[c, d]$. Then $\Lambda^{*} B V(A) \subset H B V(A)$.

Proof. Suppose $f \in \Lambda^{*} B V(A)$. Obviously $f(a, \cdot)$ and $f(\cdot, c)$ are in $H B V$. Let $\left\{I_{i}\right\}_{i=1}^{N}$ and $\left\{J_{j}\right\}_{j=1}^{M}$ be systems of nonoverlapping intervals in $[a, b]$ and $[c, d]$ respectively, and let $\Delta_{i, j}=\left|f\left(I_{i} \times J_{j}\right)\right|$. We enumerate the pairs $(i, j), i \in[1, N]$ and $j \in[1, M]$, as follows: assign 1 to $(1,1)$, and 2 and 3 to $(1,2)$ and $(2,1)$. Next we enumerate the $(i, j)$ such that $i \cdot j=3$ in any order, and so on. Let $\mu(i, j)$ denote the index corresponding to $(i, j)$. For a given $n$, the number of $(i, j)$ with $\mu(i, j) \geq 1$ and $i \cdot j \leq n$ is not greater than

$$
\sum_{i=1}^{n} \frac{n}{i} \leq n \ln (n+1)
$$

implying that, for these pairs, $\mu(i, j) \leq n \ln (n+1)$, and so

$$
\lambda_{\mu(i, j)} \leq \frac{n \ln (n+1)}{\ln (n \ln (n+1)+1)} \leq 2 n .
$$

Thus, if $i \cdot j=n$, we have

$$
\frac{\Delta_{i, j}}{i \cdot j} \leq \frac{2 \Delta_{i, j}}{\lambda_{\mu(i, j)}}
$$

for all $(i, j)$. Thus

$$
\sum_{i, j=1}^{i=N} \frac{\Delta_{i, j}}{i \cdot j} \leq 2 \sum_{i, j=1}^{i=N} \frac{\Delta_{i, j}}{\lambda_{\mu(i, j)}} \leq V_{\Lambda^{*}}(f),
$$

which establishes Lemma 3.

Remark 3. It is easily seen that $\Lambda^{*} B V(A)$ is a Banach space with $\|f\|_{\Lambda^{*}}$, as given in Definition 3(iii), as norm.

We turn now to the proof of Theorem 3.

Proof. Let $N$ be a positive integer and $M=[N / 2]$. For positive integers $m$ and $n$ such that $m \cdot n \leq M$, let

$$
A_{m n}=\left[\frac{\pi(m-1)}{N+\frac{1}{2}}, \frac{\pi m}{N+\frac{1}{2}}\right) \times\left[\frac{\pi(n-1)}{N+\frac{1}{2}}, \frac{\pi n}{N+\frac{1}{2}}\right)
$$

and set

$$
g_{N}(x, y)=\sum_{m \cdot n \leq M}(-1)^{m+n} \chi_{A_{m n}}(x, y) .
$$

If $A$ is a closed rectangle with sides parallel to the axes, then $g_{n}(A) \neq 0$ only when $A \cap A_{m n}$ contains exactly one vertex of A. Since the number of $A_{m n}$ is not greater than $M \ln (M+1)$, we have

$$
V_{\Lambda^{*}}\left(g_{n}\right) \leq \sum_{r=1}^{4 M \ln (M+1)} \frac{\ln (r+1)}{r \xi_{r}}=o\left(\ln ^{2}(M+1)\right),
$$

and so

$$
\left\|g_{N}\right\|_{\Lambda^{*}}=o\left(\ln ^{2}(M+1)\right)=\eta_{N} \ln ^{2}(M+1),
$$


where $\eta_{N}=o(1)$ as $N \rightarrow \infty$, and, if

$$
h_{N}=\frac{g_{N}}{\eta_{N} \ln ^{2}(M+1)},
$$

then $\left\{\left\|h_{N}\right\|_{\Lambda^{*}}\right\}$ is bounded.

If we now consider the square partial sums of the Fourier series of $h_{n}$ at $(0,0)$, we have

$$
\begin{aligned}
\pi^{2} S_{N N}\left[h_{N} ;(0,0)\right] & =\frac{1}{\eta_{N} \ln ^{2}(M+1)} \sum_{m \cdot n \leq M}(-1)^{m+n} \iint_{A_{m n}} D_{N}(s) D_{N}(t) d s d t \\
& \geq \frac{4}{\eta_{N} \ln ^{2}(M+1) \pi^{2}} \sum_{m \cdot n \leq M} \frac{1}{m \cdot n} \geq C \frac{1}{\eta_{N}} \frac{\ln ^{2}(M+1)}{\ln ^{2}(M+1)} \rightarrow \infty
\end{aligned}
$$

as $N \rightarrow \infty, C$ being an absolute constant. Applying the Banach-Steinhaus theorem, we see that there must be an $f \in \Lambda^{*} B V$ such that $\left\{S_{N N}[f ;(0,0)]\right\}$ diverges unboundedly.

\section{REFERENCES}

1. M. Avdispahić, Concepts of generalized bounded variation and the theory of Fourier series. Internat. J. Math. Math. Sci. 9 (1986), no. 2, 223-244. MR88c:42001

2. L. A. D'Antonio, D. Waterman, A summability method for Fourier series of functions of generalized bounded variation. Analysis 17 (1997), no. 2-3, 287-299. MR99h:42008

3. M. I. Dyachenko, Waterman classes and spherical partial sums of double Fourier series. Anal. Math. 21 (1995), no. 1, 3-21. MR97m:42009

4. C. Goffman, D. Waterman, The localization principle for double Fourier series. Studia Math. $69(1980 / 81)$, no. 1, 41-57. MR82d:42010

5. A. A. Saakyan, On the convergence of double Fourier series of functions of bounded harmonic variation. (Russian) Izv. Akad. Nauk Armyan. SSR Ser. Mat. 21 (1986), no. 6, 517-529; English transl., Soviet J. Contemp. Math. Anal. 21 (1986), no. 6, 1-13. MR88j:42017

6. A. I. Sablin, $\Lambda$-variation and Fourier series. (Russian) Izv. Vyssh. Uchebn. Zaved. Mat. 1987, no. 10, 66-68; English transl., Soviet Math. (Iz. VUZ) 31 (1987), no. 10, 87-90. MR89c:42008

7. D. Waterman, On convergence of Fourier series of functions of generalized bounded variation. Collection of articles honoring the completion by Antoni Zygmund of 50 years of scientific activity. II. Studia Math. 44 (1972), 107-117. MR 46:9623

8. Math. 54 (1975/76), no. 1, 87-95 MF 53:6212

9.

10. Fourier series of functions of $\Lambda$-bounded variation. Proc. Amer. Math. Soc. 74 (1979), no. 1, 119-123. MR80j:42010

11. 986. MR2000k:40006

Professor of the Chair of Theory of Functions and Functional Analysis, Department of Mathematics and Mechanics, Moscow State University, Vorobyevi Gori, GZ, Moscow, Russia 119992

E-mail address: dyach@mail.ru

Research Professor, Florida Atlantic University (Professor Emeritus, Syracuse University), 7739 Majestic Palm Drive, Boynton Beach, Florida 33437

E-mail address: fourier@adelphia.net 